\title{
Hemodynamic Interactions in the Eye: A Review
}

\author{
S. Mojtaba Golzan a Alberto Avolio ${ }^{a}$ Stuart L. Graham ${ }^{a}$ b \\ ${ }^{a}$ Australian School of Advanced Medicine, Macquarie University and ${ }^{b}$ Sydney University, Sydney, N.S.W., Australia
}

\begin{abstract}
Key Words
Spontaneous retinal venous pulsatility $\cdot$ Intraocular pressure $\cdot$ Cerebrospinal fluid pressure
\end{abstract}

\begin{abstract}
The ocular circulation provides readily visible information about the state of the systemic circulation, as well as being potentially of relevance to the pathogenesis of ocular disorders such as glaucoma. The interaction between intraocular pressure, retinal vessels and cerebrospinal fluid pressure located at the retrolaminar portion of the eye has been of great interest for both ophthalmic and neurological clinicians and researchers. Understanding the relationship between these physiological parameters can explain phenomena such as spontaneous retinal venous pulsatility, and characterize the effects of the translaminar pressure gradient. It may be feasible to use measurable changes in venous pulsatility to enhance clinical assessment in different diseases. In this article we review recent findings on ocular hemodynamics and the relevance of these parameters in the diagnosis of ophthalmic and neurological diseases.
\end{abstract}

Copyright $\odot 2012$ S. Karger AG, Basel

\section{Introduction}

The intraocular space is in very close proximity to the brain anatomically. The dura of the brain extends as a sheath around the optic nerve and connects with poste- rior sclera around the nerve head. The cerebrospinal fluid (CSF) flows through the optic nerve subarachnoid space around the optic nerve within this sheath. When CSF pressure (CSFp) rises it also rises inside the optic nerve sheath, transmitting this pressure to the nerve head. The central retinal artery and vein travel through the sheath and pass, along with the optic nerve fibers, into the eye [1].

The tissue of the optic disc is more distensible than the surrounding sclera and, therefore, with high CSFp it swells into the cavity of the eye. Raised CSFp can be assessed by the extent of optic disc edema $[1,2]$. It has been shown that a relationship between retinal venous caliber, intraocular pressure (IOP) and CSFp exists, with the degree of retinal venous caliber being associated with the translaminar pressure gradient (i.e. IOP-CSFp) [3].

There are a large number of publications surrounding this topic. In this article we review findings on the relationship between the different hemodynamic structures influencing the ocular circulation.

\section{Systemic Blood Pressure, Retinal Vasculature, Intraocular Pressure and Cerebrospinal Fluid Pressure}

IOP is measured using tonometry as the transcorneal pressure gradient between IOP and the atmosphere. The normal mean IOP is around $14 \mathrm{~mm} \mathrm{Hg}$ [4]. The Beijing Eye Study [5] measured the IOP of 3,253 subjects along

\section{KARGER \\ Fax +41613061234 \\ E-Mail karger@karger.ch}

www.karger.com
(C) 2012 S. Karger AG, Basel

$0030-3755 / 12 / 2284-0214 \$ 38.00 / 0$

Accessible online at:

www.karger.com/oph
S. Mojtaba Golzan

Australian School of Advanced Medicine

Macquarie University

Sydney, NSW 2109 (Australia)

Tel. +61 29812 3575, E-Mail mojtaba.golzan@mq.edu.au 
with blood pressure (BP). They reported a significant correlation between IOP and Diastolic BP $(\mathrm{p}<0.01)$. Different studies have reported a positive correlation between IOP and BP $[6,7]$. The Beaver Dam Eye Study [8] reports an increase of $0.21 \mathrm{~mm} \mathrm{Hg}$ in IOP for an increase of 10 $\mathrm{mm} \mathrm{Hg}$ in systolic BP. In relation to glaucoma, links to both hypertension and hypotension have been reported. In the Blue Mountains Eye Study [9], hypertension was associated with open angle glaucoma. The odds ratio for open angle glaucoma per standard deviation increase of systolic BP was reported to be 1.12 in the Rotterdam Eye Study [10]. Low systolic BP is reported to be a predictor for glaucoma progression [11, 12]. Twenty-four-hour ambulatory BP monitoring has reported lower nocturnal BP associated with glaucoma and progressive damage [13$15]$.

In several large epidemiological studies, lower ocular perfusion pressure (the difference between IOP and mean arterial BP) has been found to be a risk factor for glaucoma. The Early Manifest Glaucoma Trial [11] recently reported new baseline predictors for the progression of glaucoma. These included lower ocular systolic perfusion pressure in all patients, cardiovascular disease history in patients with higher baseline IOP and lower systolic BP in patients with lower baseline IOP.

\section{Animal Studies}

A study on owl monkeys [16] showed a parallel increase of orbital venous pressure along with increased CSFp. This study suggested that the pressure in the central retinal vein increases when the pressure in the subarachnoid space of the optic nerve sheath rises above the normal venous pressure. A report by Hedges and Zaren [17] on cats suggests that optic nerve subarachnoid space pressure is equal to intracranial CSFp at the same vertical level. The study demonstrated a parallel change in optic nerve tissue pressure, elevated CSFp and systemic arterial pressure. It also showed that systemic BP has a direct affect on CSFp and optic nerve tissue pressure.

Simultaneous measurement of IOP, central aortic pressure, retinal arterial pressure and retinal venous pressure (RVP) was studied in 12 cats [18]. The study showed retinal arterial pressure was 30\% lower than systemic arterial pressure. Retinal venous pressure was reported to be $10 \mathrm{~mm} \mathrm{Hg}$ higher than IOP. The authors suggested the existence of a high resistance segment between the retinal and systemic vein as the possible cause of high RVP. Another study by this group investigated the affect of high IOP on vascular pressures of the cat retina
[19]. Retinal artery and venous pressure along with IOP was measured in the retina of 8 cats using a micropipette. The study reported a significant correlation between the transmural pressure $\mathrm{P}_{\mathrm{tv}}(\mathrm{RVP}-\mathrm{IOP})$ and IOP. The relation was defined as: $\mathrm{P}_{\mathrm{tv}}=9.12-0.0964$ IOP.

The translaminar pressure gradient was investigated by Morgan et al. [20]. Micropipettes were inserted into the optic disc of anesthetized dogs. They recorded lateral ventricle CSFp, IOP and arterial BP simultaneously at baseline and with raised CSFp. They showed a dependency of retrolaminar tissue pressure on surrounding CSFp and independent of IOP. They also reported that the CSFp in the optic nerve sheath is identical to the lateral ventricle CSFp, confirming hydrostatic continuity of CSF along the optic nerve subarachnoid space.

Westlake et al. [21] investigated the Starling resistor theory of venous outflow from the eye in 6 pig eyes using servo-nulling micropuncture technique. IOP, RVP and CSFp were recorded and monitored. RVP was shown to be highly correlated with IOP $\left(\mathrm{r}^{2}=0.92\right)$ with no significant correlation found between CSFp and retinal venous transmural pressure. The study suggested that IOP increases in response to a reduction of ocular blood flow which in return will increase outflow resistance allowing RVP to follow changes in IOP. Other findings of this study show a significantly lower transmural venous pressure on the optic disc (and sometimes negative pressures) compared to downstream within the vessels. This is consistent with a Starling resistor model of venous outflow.

A more recent study [22] reported the translaminar pressure changes in rats. The study monitored CSFp and IOP for $24 \mathrm{~h}$ and their reaction to light. During the light or dark period the CSFp did not change significantly, whereas the IOP was higher in dark periods altering the translaminar pressure gradient.

\section{Human Studies}

Sajjadi et al. [23] studied the relation between IOP and intracranial pressure (same as CSFp) in 50 patients. IOP was measured using Schiotz tonometer and CSFp was measured by lumbar puncture technique. The CSFp range was $5.15-47.8 \mathrm{~mm} \mathrm{Hg}$ and the IOP 7.17-36.9 $\mathrm{mm}$ $\mathrm{Hg}$. The results showed a significant correlation $(\mathrm{p}<$ $0.001 ; \mathrm{r}=0.955 ; \mathrm{n}=50$ ) between IOP and CSFp. Querfurth et al. [24] reported on the relationship between ocular blood flow velocities and pulsatility to CSFp. They tested 16 patients (32 eyes) with chronic intracranial hypertension and 16 normal age-matched subjects using color Doppler imaging. The results show a sig- 
nificantly lower arterial flow velocity in intracranial hypertension subjects compared to normals. The results also indicate an increase in vascular resistance due to elevated CSFp.

In a recent study [25] where the translamina cribrosa pressure gradient was determined, 17 ocular hypertensive and 71 normal subjects were studied and the authors designated IOP-CSFp as the translamina cribrosa pressure difference. IOP was measured using Goldmann applanation tonometry and lumbar puncture was performed to record opening CSFp. IOP and CSFp were both significantly higher in ocular hypertensives compared to normals. The study also reports a high correlation between CSFp, IOP and arterial BP in both groups ( $\mathrm{p}<0.01)$. A similar study [26] on a large population compared CSFp in glaucomatous and nonglaucomatous patients. From this population, 28 glaucomatous and 49 nonglaucomatous subjects were studied. CSFp was measured by means of lumbar puncture. The study reported a significantly lower CSFp in patients with glaucoma compared to others. The authors also observed a significant association between cup-to-disc ratio and low CSFp.

Sheeran et al. [27] disputes the possibility that changes in IOP can be a predictor of CFSp changes. They studied 31 patients who had recording catheters inserted into the brain (either brain tissue or ventricles). IOP was recorded using a noncontact tonometer (Keeler Pulsair air impulse tonometer). They observed a mean correlation of $\mathrm{r}^{2}=0.46$ between IOP and CSFp. They also showed that the rate of CSFp increase per mm $\mathrm{Hg}$ of IOP could vary between subjects, thus the rise in CSFp per $\mathrm{mm} \mathrm{Hg}$ of IOP was not significant between patients. They concluded that IOP and CSFp correlate significantly within each individual but vary notably between patients. Therefore, changes in IOP alone were an insensitive indicator of CSFp changes. Czarnik et al. [28] confirmed the findings of Sheeran et al. [27] by measuring IOP, CSFp and mean arterial pressure simultaneously in 40 patients with brain injuries who were admitted to hospital. The studies show only a significant correlation between IOP and mean arterial pressure, but not with CSFp.

A hypothesis upon the effect of CSFp on the pathogenesis of glaucoma has been reported by Yang et al. [29]. Based on a review of the literature, the authors postulated that glaucoma is not solely an IOP-related disease. Fluctuations of CSF in the subarachnoid space of the optic nerve may contribute to the optic neuropathy of glaucoma.

\section{Spontaneous Retinal Venous Pulsatility}

\section{Clinical Significance of Spontaneous Retinal Venous Pulsatility}

The spontaneous pulsation of retinal veins has been observed since the late 19th century [30]. It can be directly visualized with an ophthalmoscope. The phenomenon was first explained as a result of retinal venous compression caused by IOP during systolic blood influx [30]. Later in the 20th century a loss of these pulsations during intracranial hypertension was reported [31]. Similar results were observed by other researchers $[32,33]$. Therefore, it would be of great clinical significance if one could measure the intracranial pressure and the behavior of the retinal pulse following changes of the ocular pressure. Accordingly, spontaneous retinal venous pulsatility (SRVP) has attracted the interest of many researchers; however, no comprehensive explanation has been raised from different studies.

A combination of theories has been reported to possibly describe the nature of SRVP. An early study by Levin [34] examined SRVP in 218 subjects using an ophthalmoscope and CSFp measured by lumbar puncture. His study showed that SRVP was present in $87.6 \%$ of 146 subjects with normal CSFp and 100\% absent in 33 subjects with raised CSFp. A similar study on 65 subjects [35] confirms Levin's findings and suggests that the presence of SRVP is a significant clinical sign of normal intracranial pressure.

The venous collapse phenomenon was studied by a physically built technical model [36]. IOP, central retinal vein transmural pressure and the overall perfusion was investigated in association with the venous collapse. The authors measured the venous outflow pressure (VOP) directly by pressure catheters or indirectly by raising IOP and registering the pressure at which the vein began to collapse. The study showed a relationship between venous collapse and VOP which has been proven to be clinically significant. Furthermore, the authors demonstrated a strong relation between CSFp and VOP.

Elliot [37] described in detail the findings and theory of a French author, Bailliart [38], about the nature of SRVP. Elliot believed that for a given cardiac cycle, IOP exceeds the pressure inside the central retinal vein (i.e. RVP) during ventricular systole hence forcing the vein to collapse. During diastole RVP exceeds IOP resulting in the reexpansion of the vein. According to this theory, two explanations were put forward, firstly that RVP could be higher or lower than IOP and secondly that pressure fluctuations in either one of these parameters does not necessarily transmit to another. 
Elliot believed that a large difference between IOP and RVP in some individuals prevented the fluctuation in either to alter the pressure gradient direction, leading to no pulsation. He suggested that if RVP exceeds IOP substantially, SRVP could be induced by pressing on the eye and elevating IOP. However, if the case is reversed (i.e. IOP exceeds RVP), SRVP cannot be induced manually.

A more recent explanation for the nature of SRVP is provided by Levine [39]. He criticized the theory of Elliot and Bailliart in two areas: firstly, that IOP fluctuations must be transmitted to RVP. He explains that a rise in IOP would not alter the capillaries and veins as long as these vessels have rigid walls or, alternatively, the blood inflow rate is exactly equal to the blood outflow rate in ocular veins. Secondly, recent findings $[18,19,40]$ have all measured RVP and IOP at several levels of IOP and shown that RVP consistently exceeds IOP.

On this basis Levine proposed a constant input/variable output theory. This was based on the following assumptions: (1) the RVP is subject to intraocular space pressure (i.e. IOP); the central retinal vein exits the eye through the optic disc and passes through the lamina cribrosa. This leads to RVP being subjected to CSFp on the retrolaminar portion. (2) Blood flowing from capillaries into veins is constant and remains pulsatile as it reaches the optic disc which is then subjected to a translaminar pressure gradient resulting in SRVP. During systole an increase in exiting blood flow occurs by depleting blood volume from the vein, hence causing it to collapse. During diastole, due to constant inflow, a decrease in exiting blood flow will lead to an increase in venous blood volume, thereby forcing the vein to expand.

Levine proposed a mathematical model for venous pulsation. The model states that venous pulsation has maxima at the exit point, and amplitudes decrease at the distal veins where the amplitude of pulse is relatively small compared to its diameter. Furthermore, the theory explained that IOP fluctuates in phase with RVP. It is also consistent with experimental data stating that RVP always exceeds IOP.

Finally, according to the constant input/variable output theory, during an increase in CSFp, the CSF pulsations and the mean CSFp rise [41] and approach the intraocular pulse pressure (IOPP), resulting in a zero intravascular pressure gradient over the prelaminar and retrolaminar optic nerve, leading to cessation of the retinal venous pulsation. These findings have been confirmed by other studies [42-45]. The constant input/variable output theory also offers a possible explanation on SRVP variability from one normal individual to another.

Hemodynamic Interactions in the Eye:

A Review
This is explained by the high degree of dependency of translaminar blood flow on the difference between IOP and CSFp amplitude in each cardiac cycle.

Parsa [44] explains SRVP occurrence by the excess of IOP over RVP during systole, hence forcing the vein to collapse. He suggests that lowering IOP to minimize the pressure gradient between the intraocular and retrolaminar space will drop SRVP and could be an indicator of CSFp. This explanation is supported by Jacks and Miller [43]. They reported that SRVP is a result of variation in the pressure gradient along the central retinal vein as it travels through the lamina cribrosa. Moreover, the pressure gradient variation is a result of differences in pulse pressure between intraocular space and CSF. They concluded that SRVP cessation is explained by equal intracranial pulse pressure and IOPP during intracranial hypertension and, therefore, SRVP could be a good clinical indicator of CSFp. However, the cessation of SRVP can be caused by other factors than elevated CSFp. McCulley et al. [46] investigated the effects of optic disc edema on SRVP. They studied 20 subjects with unilateral optic disc edema without elevated CSFp. SRVP was present in $5 \%$ of the involved eyes and $60 \%$ of the uninvolved eyes. Their study discusses the theory that loss of SRVP may not necessarily indicate high CSFp; however, the study was limited by the fact that CSFp was only measured in some of the subjects.

Donnelly and Subramanian [42] studied the relation between IOPP and SRVP. They examined 47 subjects without any symptoms of raised CSFp. They measured IOP using applanation tonometry and IOPP using the Pascal dynamic tonometer. They observed a $91.5 \%$ presence of SRVP in their subjects. SRVP was detected in eyes with an IOPP greater than $1.2 \mathrm{~mm} \mathrm{Hg}$. The final conclusion of this study was that the absence of SRVP in at least one eye with IOPP greater than $1.2 \mathrm{~mm} \mathrm{Hg}$ could be a sign of elevated CSFp. This cutoff point was questioned by Nowroozzadeh and Saki [47], who argued that CSFp pulse may have the same amount of effect on SRVP as IOPP. Therefore, defining a cutoff point of $1.2 \mathrm{~mm} \mathrm{Hg}$ may not necessarily be of clinical relevance.

One study [48] disagrees with the fact that venous collapse is induced only by IOP and is in phase with IOP. The authors hypothesize that SRVP is probably a flow-induced phenomenon rather than a vascular transmural gradient pressure. The study is in major conflict with other reports.

Finally, the timing and IOP induction of SRVP have been investigated [49]. SRVP with IOP fluctuations at each individual cardiac cycle was studied in 10 subjects. 
Venous collapse was recorded $40 \mathrm{~ms}$ after minimum IOP whereas venous maximum expansion was registered $5 \mathrm{~ms}$ after maximum IOP occurred. This is intriguing as previous studies suggested that venous collapse occurs after maximum IOP. The study concluded that IOP is not the only factor in venous collapse and that CSF pulse pressure is equivalent to IOP pulse as a contributing factor.

\section{SRVP in Glaucoma}

Harder and Jonas [50] observed SRVP in 90\% of 384 subjects tested for SRVP presence. They recommended that SRVP can be a good indicator of CSFp and that if a patient presents to a clinic with a lack of SRVP he has a $90 \%$ chance of an abnormal clinical situation.

The frequency of SRVP presence is also reported by Morgan et al. [51]. They compared SRVP in 3 groups of normals, glaucomatous eyes and glaucoma suspects. They reported an SRVP presence of 98, 54 and 75\% in these groups, respectively. Morgan et al. [51] explain the significant drop of SRVP in glaucoma subjects with three possible theories. Firstly, an increased CSFp can lead to a low intravascular pressure gradient between IOP and CSFp; however, they did not observe any signs of raised CSFp such as papilledema in these subjects. Secondly, they suggest that upstream vascular changes may be a factor, arterial BP may cause an upstream vascular pressure increase, but they could not measure this pressure in their subjects to support this explanation. Finally, they suggest an increase in downstream resistance [52].

Translaminar pressure gradient was investigated in glaucoma patients with normal and elevated IOP [45]. There was a significantly higher translaminar pressure gradient in the first group compared to the latter. This is observed due to abnormally lower CSFp in subjects with normal IOP readings. The study concluded that a high translaminar pressure gradient may be a vital factor in the pathogenesis of glaucoma. Golzan et al. [3] analyzed the SRVP amplitude in regard to IOP manipulation. They showed that lowering IOP will decrease SRVP amplitude. They also demonstrated that changes in SRVP were independent of heart rate and BP changes.

\section{Noninvasive Assessment of CSFp}

Currently, CSFp monitoring is achieved using either sensors implanted within the cranium or external sensors connected to the measurement site in the cranium with a fluid-filled catheter. Both approaches are invasive, generating a risk of intracranial infection, and require neurosurgical expertise for the implantation.

Clinical techniques for the estimation of CSFp are very limited and include assessment of the level of consciousness on neurological examination, ophthalmoscopic examination of the optic disc for papilledema, and palpation of the fontanelles and skull sutures in infants.

The central retinal vein exits the eye through the optic disc, passes through the optic nerve and its sheath, draining into the superior ophthalmic vein. Since pressure within the central retinal vein has to overcome the optic nerve sheath pressure it has to be greater than this pressure and thus higher than CSFp. Therefore, examination of the optic nerve sheath, central retinal arteries and hemodynamic veins, and their relationship to the inner ocular circulation, may give an insight into CSFp values. To this extent, different techniques including examination of the optic nerve sheath diameter (ONSD), ophthalmodynamometry, ultrasound and Doppler have been used to assess CSFp noninvasively through the eye.

To determine the use of handheld IOP tonometers in the assessment of elevated CSFp, Muchnok et al. [53] tested 46 patients. IOP was measured in the supine position using Tono-Pen XL before performing lumbar puncture to register CSFp. A cutoff pressure of $20 \mathrm{~cm} \mathrm{H}_{2} \mathrm{O}$ was used to determine elevated IOP and opening CSFp. Results show a less significant correlation between elevated IOP and elevated opening pressure (sensitivity $24 \%$, specificity 63\%). The study concluded that handheld tonometry has low sensitivity to assess CSFp.

Another approach to assess CSFp is ophthalmodynamometry. The implementation of this test starts with recording baseline IOP. The IOP is then increased until a pressure is reached for which the vein collapses. This pressure is recorded as VOP. The pressure reading (VOP) from the ophthalmodynamometer is converted to $\mathrm{mm}$ $\mathrm{Hg}$ and added to baseline IOP [54]. Firsching et al. [55] studied 22 subjects who had surgical procedures to place intraventricular catheters in their brain to measure CSFp. Ophthalmodynamometry was performed on these subjects in order to record VOP. Their results demonstrate a significant correlation between VOP and CSFp ( $\mathrm{p}<$ 0.001 ). The relationship between these two parameters was described as: CSFp $=0.903$ VOP -8.87 .

The study suggested that ophthalmodynamometry could be a reliable source for absolute CSFp estimations, with its major limitation being the lack of ability to monitor CSFp continuously. Nevertheless, this technique could be applied to evaluate disorders associated with elevated CSFp such as brain trauma, hydrocephalus and 
space-occupying lesions. Most recently the same research group [56] expanded their sample size to 102 subjects and reported a similar significant correlation between VOP and CSFp. Their results showed that an increased VOP showed a probability of $84.2 \%$ of elevated CSFp, whereas this value was $92.8 \%$ for normal VOP and CSFp.

Jonas et al. [57] reported on a case diagnosed with idiopathic intracranial hypertension. They performed ophthalmodynamometry to evaluate VOP. Lumbar puncture was also carried out to record CSFp. They reported a relationship between VOP and CSFp increase. Motschmann et al. [58] investigated 31 subjects with intraventricular CSF catheter placement. VOP was measured using ophthalmodynamometry techniques. They demonstrated a significant correlation between VOP and CSFp $(r=0.968)$. The study concluded that ophthalmodynamometry is a reliable means of noninvasive CSFp assessment.

Ophthalmodynamometry has also been used on 6 dogs to define relations between systemic and ophthalmodynamometric pressures (ODP) [59]. ODP was performed on central retinal arteries. Diastolic ODP was registered as the lowest IOP to force central retinal arteries to pulsate. Furthermore, IOP was increased until continuous central retinal artery collapse occurred. This pressure was designated as systolic ODP. Results show that diastole ODP occurred at aortic diastole, but ODP systole was registered at $96 \%$ of aortic systolic pressure.

Ultrasound is another approach which has been widely used to assess CSFp noninvasively. Orbit ultrasound probes use a frequency range of $5-10.5 \mathrm{MHz}$ to monitor the eye and vessels [60, 61]. A study [62] on cadavers using ultrasound demonstrated that elevated CSFp can increase the ONSD by more than $50 \%$. They were also able to show a relationship between the ONSD and lumbar CSFp [63]. Tamburelli et al. [64] demonstrated an expansion of the ONSD when the diastolic CSFp is greater than $14 \mathrm{~mm} \mathrm{Hg}$. For greater pressures they observed a linear relationship between ONSD enlargement and CSFp elevation. Using ultrasound they could detect CSFp greater than $15 \mathrm{~mm} \mathrm{Hg}$ with a sensitivity and specificity of 88 and $90 \%$, respectively.

Several other studies have established a correlation between the ONSD and elevated CSFp using ultrasound [65-70]. Tsung et al. [71] measured the ONSD with a $7-\mathrm{MHz}$ probe as $5 \mathrm{~mm}$ in adults, $4.5 \mathrm{~mm}$ in children and $4 \mathrm{~mm}$ in infants $[72,73]$. The study reported 3 cases with head trauma. Clinical examinations suggested a suspected elevation of CSFp in 1 of the 3 subjects with the ONSD reading confirmed at $4.4 \mathrm{~mm}$ in the 8 -month-old infant. The other cases had ONSD within the normal range.

Similar studies have been performed on children. One study [74] demonstrated a significant correlation between ONSD and suspected CSFp elevation which showed on CT or transcranial ultrasound. Le et al. [75] showed a sensitivity and specificity of 83 and $38 \%$, respectively, using opening lumbar pressure for noninvasive CSFp estimation. These values were reported as 100 and $86 \%$, respectively, in another study [76] for CSFp assessment from CT images.

Color Doppler has also been used to evaluate CSFp noninvasively. While the technique uses ultrasound, a lower pressure must be applied to the eyeball to avoid a decrease in blood flow velocity [77]. Querfurth et al. [24, 78 ] investigated VOP in association with invasive CSFp. Furthermore, they recorded central retina and ophthalmic arterial flow using the Doppler imaging technique. A direct linear relation between VOP and CSFp was reported. Central retinal artery and ophthalmic artery pulsatility dropped inversely with CSFp. The study suggested that VOP evaluation in conjunction with retinal arterial parameters may provide an approach to assess CSFp noninvasively. A further study [79] showed that systolic blood flow velocity in the central retinal artery is significantly lower in children with elevated CSFp.

\section{Conclusions}

In this article we reviewed different aspects of ocular hemodynamics and the interaction with CSFp. Most studies suggest that a strong correlation between ocular physiological structures and there pulsatility exists. Specifically, SRVP can be utilized as a marker for CSFp assessment. Although further studies are required to better define the factors involved in CSFp estimation, dynamic assessment of SRVP has been shown to be affected directly by IOP and CSFp. Furthermore, SRVP in association with the arterial BP waveform can be used to noninvasively estimate the CSFp waveform [80].

Our studies confirmed that the adjacent hemiretinal vein pulsation near the optic disc has the same characteristics as the SRVP on the optic disc. A higher IOP can be achieved transiently by applying digital pressure on the eye (or with ophthalmodynamometry). We confirmed with dynamic recording [3] that digital pressure increases SRVP amplitude dramatically. It is interesting to note that SRVP is reported to be actually reduced in glaucoma subjects [51] and that loss of SRVP may be associated with 
glaucoma progression [81]. It may be that there is raised venous pressure or the translaminar pressure gradient is reduced for other reasons in this disease.

Studies published on ocular hemodynamics have recently increased our knowledge of the ocular circulation, but the interactions occurring at the optic nerve head between the different pressurized compartments are com- plex. Further studies may lead to the utilization of vascular pulse characteristics in the clinical assessment of ophthalmic and neurological abnormalities.

\section{Disclosure Statement}

The authors declare that they have no conflicts of interest.

\section{References}

1 Guyton, AC, Hall JE: Textbook of Medical Physiology. Philadelphia, Elsevier Saunders, 2006.

2 Kaufman PL, Adler FH: Adler's Physiology of the Eye: Clinical Application. St Louis, Mosby, 2003.

-3 Golzan SM, Graham SL, Leaney J, Avolio A: Dynamic association between intraocular pressure and spontaneous pulsations of retinal veins. Curr Eye Res 2011;36:53-59.

-4 Hollows FC, Graham PA: Intra-ocular pressure, glaucoma, and glaucoma suspects in a defined population. Br J Ophthalmol 1966; 50:570-586.

$5 \mathrm{Xu} \mathrm{L}$, Wang $\mathrm{H}$, Wang Y, Jonas JB: Intraocular pressure correlated with arterial blood pressure: the Beijing Eye Study. Am J Ophthalmol 2007;144:461-462.

-6 Wu S-Y, Nemesure B, Hennis A, Leske MC: Nine-year changes in intraocular pressure: the Barbados Eye Studies. Arch Ophthalmol 2006;124:1631-1636.

7 Yip JLY, Aung T, Wong T-Y, Machin D, Khaw PT, Khaw K-T, Seah S, Foster PJ: Socioeconomic status, systolic blood pressure and intraocular pressure: the Tanjong Pagar Study. Br J Ophthalmol 2007;91:56-61.

8 Klein BE, Klein R, Knudtson MD: Intraocular pressure and systemic blood pressure: longitudinal perspective: the Beaver Dam Eye Study. Br J Ophthalmol 2005;89:284287.

-9 Mitchell P, Lee AJ, Rochtchina E, Wang JJ: Open-angle glaucoma and systemic hypertension: the Blue Mountains Eye Study. J Glaucoma 2004;13:319-326.

10 Dielemans I, Vingerling JR, Algra D, Hofman A, Grobbee DE, de Jong PT: Primary open-angle glaucoma, intraocular pressure, and systemic blood pressure in the general elderly population. The Rotterdam Study. Ophthalmology 1995;102:54-60.

- 11 Leske MC, Heijl A, Hyman L, Bengtsson B, Dong L, Yang Z: Predictors of long-term progression in the early manifest glaucoma trial. Ophthalmology 2007;114:1965-1972.

-12 Leske MC, Wu S-Y, Hennis A, Honkanen R, Nemesure B: Risk factors for incident openangle glaucoma: the Barbados Eye Studies. Ophthalmology 2008;115:85-93.
13 Graham SL, Drance SM: Nocturnal hypotension: role in glaucoma progression. Surv Ophthalmol 1999;43(suppl 1):S10-S16.

14 Graham SL, Drance SM, Wijsman K, Douglas GR, Mikelberg FS: Ambulatory blood pressure monitoring in glaucoma. The nocturnal dip. Ophthalmology 1995;102:61-69.

15 Hayreh SS, Zimmerman MB, Podhajsky P, Alward WL: Nocturnal arterial hypotension and its role in optic nerve head and ocular ischemic disorders. Am J Ophthalmol 1994; 117:603-624.

16 Rios-Montenegro EN, Anderson DR, David NJ: Intracranial pressure and ocular hemodynamics. Arch Ophthalmol 1973;89:52-58.

17 Hedges TR, Zaren HA: The relationship of optic nerve tissue pressure to intracranial and systemic arterial pressure. Am J Ophthalmol 1973;75:90-98.

18 Glucksberg MR, Dunn R: Direct measurement of retinal microvascular pressures in the live, anesthetized cat. Microvasc Res 1993;45:158-165.

19 Attariwala R, Giebs CP, Glucksberg MR: The influence of elevated intraocular pressure on vascular pressures in the cat retina. Invest Ophthalmol Vis Sci 1994;35:1019-1025.

20 Morgan WH, Yu DY, Cooper RL, Alder VA, Cringle SJ, Constable IJ: The influence of cerebrospinal fluid pressure on the lamina cribrosa tissue pressure gradient. Invest Ophthalmol Vis Sci 1995;36:1163-1172.

21 Westlake WH, Morgan WH, Yu DY: A pilot study of in vivo venous pressures in the pig retinal circulation. Clin Experiment Ophthalmol 2001;29:167-170.

22 Lin JS, Liu JH: Circadian variations in intracranial pressure and translaminar pressure difference in Sprague-Dawley rats. Invest Ophthalmol Vis Sci 2010;51:5739-5743.

-23 Sajjadi SA, Harirchian MH, Sheikhbahaei N, Mohebbi MR, Malekmadani MH, Saberi H: The relation between intracranial and intraocular pressures: study of 50 patients. Ann Neurol 2006;59:867-870.

24 Querfurth HW, Lagrèze WD, Hedges TR, Heggerick PA: Flow velocity and pulsatility of the ocular circulation in chronic intracranial hypertension. Acta Neurol Scand 2002; 105:431-440.
25 Ren R, Zhang X, Wang N, Li B, Tian G, Jonas JB: Cerebrospinal fluid pressure in ocular hypertension. Acta Ophthalmol 2011; 89:e142-e148.

26 Berdahl JP, Allingham RR, Johnson DH: Cerebrospinal fluid pressure is decreased in primary open-angle glaucoma. Ophthalmology 2008;115:763-768.

27 Sheeran P, Bland JM, Hall GM: Intraocular pressure changes and alterations in intracranial pressure. Lancet 2000;355:899.

28 Czarnik T, Gawda R, Kolodziej W, Latka D, Sznajd-Weron K, Weron R: Associations between intracranial pressure, intraocular pressure and mean arterial pressure in patients with traumatic and non-traumatic brain injuries. Injury 2009;40:33-39.

29 Yang Y, Yu M, Zhu J, Chen X, Liu X: Role of cerebrospinal fluid in glaucoma: pressure and beyond. Med Hypotheses 2010;74:31-34.

30 Coccius EA: On the Application of Eye-View Mirror in Addition to Specifying a New Device. Leipzig, Muller-Leipzig, 1853, pp 3-23.

31 Baurmann M: On the origin and clinical significance of retinal venous pulse. Zusammenkunft Dtsch Ophthalmol 1925;45:5359.

32 Kahn EA, Cherry GR: The clinical importance of spontaneous retinal venous pulsation. Med Bull (Ann Arbor) 1950;16:305308.

33 Walsh TJ, Garden JW, Gallagher B: Obliteration of retinal venous pulsations during elevation of cerebrospinal-fluid pressure. Am J Ophthalmol 1969;67:954-956.

34 Levin BE: The clinical significance of spontaneous pulsations of the retinal vein. Arch Neurol 1978;35:37-40.

35 LoZito JC: Retinal spontaneous venous pulsations. Arch Neurol 1978;35:478.

36 Meyer-Schwickerath R, Kleinwächter T, Firsching R, Papenfuss HD: Central retinal venous outflow pressure. Graefes Arch Clin Exp Ophthalmol 1995;233:783-788.

37 Elliot RH: The retinal pulse. Br J Ophthalmol 1921;5:481-500.

38 Bailliart P: La circulation veineuse rétinienne. Ann Ocul (Paris) 1918;155:453-472.

39 Levine DN: Spontaneous pulsation of the retinal veins. Microvasc Res 1998;56:154165 . 
-40 Morgan WH, Yu DY, Cooper RL, Alder VA, Cringle SJ, Constable IJ: Retinal artery and vein pressures in the dog and their relationship to aortic, intraocular, and cerebrospinal fluid pressures. Microvasc Res 1997;53:211-221.

-41 Dardenne G, Dereymaeker A, Lacheron JM: Cerebrospinal fluid pressure and pulsatility. An experimental study of circulatory and respiratory influences in normal and hydrocephalic dogs. Eur Neurol 1969;2:193-216.

-42 Donnelly SJ, Subramanian PS: Relationship of intraocular pulse pressure and spontaneous venous pulsations. Am J Ophthalmol 2009; 147:51-55.

-43 Jacks AS, Miller NR: Spontaneous retinal venous pulsation: aetiology and significance. J Neurol Neurosurg Psychiatry 2003;74:7-9.

44 Parsa CF: Spontaneous venous pulsations should be monitored during glaucoma therapy. Br J Ophthalmol 2002;86:1187.

-45 Ren R, Jonas JB, Tian G, Zhen Y, Ma K, Li S, Wang H, Li B, Zhang X, Wang N: Cerebrospinal fluid pressure in glaucoma: a prospective study. Ophthalmology 2010;117:259-266.

-46 McCulley TJ, Lam BL, Bose S, Feuer WJ: The effect of optic disk edema on spontaneous venous pulsations. Am J Ophthalmol 2003; 135:706-708.

-47 Nowroozzadeh MH, Saki S: Intraocular pulse pressure affects the sensitivity and specificity of spontaneous venous pulsation test. Am J Ophthalmol 2009;147:945, author reply 945-946.

48 Attariwala R, Glucksberg MR, Aitken V, Cuzzani OE, Gimbel HV: Evaluation of the spontaneous retinal vein pulse. Proceedings of the 1st Joint BMES/EMBS Conference, Atlanta, October 1999.

-49 Kain S, Morgan WH, Yu D-Y: New observations concerning the nature of central retinal vein pulsation. Br J Ophthalmol 2010;94: 854-857.

50 Harder B, Jonas JB: Frequency of spontaneous pulsations of the central retinal vein. $\mathrm{Br}$ J Ophthalmol 2007;91:401-402.

-51 Morgan WH, Hazelton ML, Azar SL, House $\mathrm{PH}, \mathrm{Yu}$ D-Y, Cringle SJ, Balaratnasingam C: Retinal venous pulsation in glaucoma and glaucoma suspects. Ophthalmology 2004; 111:1489-1494.

52 The Eye Disease Case-Control Study Group: Risk factors for central retinal vein occlusion. Arch Ophthalmol 1996;114:545-554.

53 Muchnok T, Deitch K, Giraldo P: Can intraocular pressure measurements be used to screen for elevated intracranial pressure in emergency department patients? J Emerg Med 2010, E-pub ahead of print.

54 Firsching R, Schütze M, Motschmann M, Behrens-Baumann W, Meyer-Schwickerath $\mathrm{R}$ : Non-invasive measurement of intracranial pressure. Lancet 1998;351:523-524.

-55 Firsching R, Schütze M, Motschmann M, Behrens-Baumann W: Venous opthalmodynamometry: a noninvasive method for assessment of intracranial pressure. J Neurosurg 2000;93:33-36.
Firsching R, Müller C, Pauli S-U, Voellger B, Röhl F-W, Behrens-Baumann W: Noninvasive assessment of intracranial pressure with venous ophthalmodynamometry. Clinical article. J Neurosurg 2011;115:371-374.

57 Jonas JB, Pfeil K, Chatzikonstantinou A, Rensch F: Ophthalmodynamometric measurement of central retinal vein pressure as surrogate of intracranial pressure in idiopathic intracranial hypertension. Graefes Arch Clin Exp Ophthalmol 2008;246:1059-1060.

58 Motschmann M, Müller C, Kuchenbecker J, Walter S, Schmitz K, Schütze M, Behrens-Baumann W, Firsching R: Ophthalmodynamometry: a reliable method for measuring intracranial pressure. Strabismus 2001;9:13-16.

-59 Morgan WH, Yu DY, Alder VA, Cringle SJ, Constable IJ: Relation between pressure determined by ophthalmodynamometry and aortic pressure in the dog. Br J Ophthalmol 1998;82:821-825.

60 Hewick SA, Fairhead AC, Culy JC, Atta HR: A comparison of $10 \mathrm{MHz}$ and $20 \mathrm{MHz}$ ultrasound probes in imaging the eye and orbit. Br J Ophthalmol 2004;88:551-555.

61 Shah S, Kimberly H, Marill K, Noble VE: Ultrasound techniques to measure the optic nerve sheath: is a specialized probe necessary? Med Sci Monit 2009;15:MT63-MT68.

62 Helmke K, Hansen HC: Fundamentals of transorbital sonographic evaluation of optic nerve sheath expansion under intracranial hypertension. I. Experimental study. Pediatr Radiol 1996;26:701-705.

63 Hansen HC, Helmke K: Validation of the optic nerve sheath response to changing cerebrospinal fluid pressure: ultrasound findings during intrathecal infusion tests. J Neurosurg 1997;87:34-40.

64 Tamburelli C, Aricle C, Mangiola A, et al: CSF dynamic parameters and changes of optic nerve diameters measured by standardized echography. Ophthalmic Echogr 1993; 13:101-109.

65 Geeraerts T, Launey Y, Martin L, Pottecher J, Vigué B, Duranteau J, Benhamou D: Ultrasonography of the optic nerve sheath may be useful for detecting raised intracranial pressure after severe brain injury. Intensive Care Med 2007;33:1704-1711.

66 Geeraerts T, Merceron S, Benhamou D, Vigué B, Duranteau J: Non-invasive assessment of intracranial pressure using ocular sonography in neurocritical care patients. Intensive Care Med 2008;34:2062-2067.

67 Kimberly HH, Shah S, Marill K, Noble V: Correlation of optic nerve sheath diameter with direct measurement of intracranial pressure. Acad Emerg Med 2008;15:201-204. 68 Moretti R, Pizzi B: Optic nerve ultrasound for detection of intracranial hypertension in intracranial hemorrhage patients: confirmation of previous findings in a different patient population. J Neurosurg Anesthesiol 2009;21:16-20.

69 Moretti R, Pizzi B, Cassini F, Vivaldi N: Reliability of optic nerve ultrasound for the eval- uation of patients with spontaneous intracranial hemorrhage. Neurocrit Care 2009; 11:406-410.

-70 Soldatos T, Karakitsos D, Chatzimichail K, Papathanasiou M, Gouliamos A, Karabinis A: Optic nerve sonography in the diagnostic evaluation of adult brain injury. Crit Care 2008; 12:R67.

71 Tsung JW, Blaivas M, Cooper A, Levick NR: A rapid noninvasive method of detecting elevated intracranial pressure using bedside ocular ultrasound: application to 3 cases of head trau$\mathrm{ma}$ in the pediatric emergency department. Pediatr Emerg Care 2005;21:94-98.

-72 Ballantyne J, Hollman AS, Hamilton R, Bradnam MS, Carachi R, Young DG, Dutton GN: Transorbital optic nerve sheath ultrasonography in normal children. Clin Radiol 1999;54:740-742.

-73 Newman WD, Hollman AS, Dutton GN, Carachi R: Measurement of optic nerve sheath diameter by ultrasound: a means of detecting acute raised intracranial pressure in hydrocephalus. Br J Ophthalmol 2002;86: 1109-1113.

74 Malayeri AA, Bavarian S, Mehdizadeh M: Sonographic evaluation of optic nerve diameter in children with raised intracranial pressure. J Ultrasound Med 2005;24:143-147.

75 Le A, Hoehn ME, Smith ME, Spentzas T, Schlappy D, Pershad J: Bedside sonographic measurement of optic nerve sheath diameter as a predictor of increased intracranial pressure in children. Ann Emerg Med 2009;53: 785-791.

-76 Beare NAV, Kampondeni S, Glover SJ, Molyneux E, Taylor TE, Harding SP, Molyneux ME: Detection of raised intracranial pressure by ultrasound measurement of optic nerve sheath diameter in African children. Trop Med Int Health 2008;13:1400-1404.

77 Tranquart F, Bergès O, Koskas P, Arsene S, Rossazza C, Pisella P-J, Pourcelot L: Color Doppler imaging of orbital vessels: personal experience and literature review. J Clin Ultrasound 2003;31:258-273.

78 Querfurth HW, Arms SW, Lichy CM, Irwin WT, Steiner T: Prediction of intracranial pressure from noninvasive transocular venous and arterial hemodynamic measurements: a pilot study. Neurocrit Care 2004;1: 183-194.

79 Miller MM, Chang T, Keating R, Crouch E, Sable C: Blood flow velocities are reduced in the optic nerve of children with elevated intracranial pressure. J Child Neurol 2009;24: 30-35.

80 Golzan SM, Kim MO, Seddighi AS, Avolio A, Graham SL: Non-invasive estimation of cerebrospinal fluid pressure waveforms by means of retinal venous pulsatility and central aortic blood pressure. Ann Biomed Eng 2012, E-pub ahead of print.

81 Graham SL, Butlin M, Lee M, Avolio AP Central blood pressure, arterial waveform analysis, and vascular risk factors in glaucoma. J Glaucoma 2012, E-pub ahead of print. 